\title{
Transfert d'énergie des neutrons rapides dans quelques matériaux radiothermoluminescents $\left(^{*}\right)$
}

\author{
F. SPURNÝ $(* *)$, R. MÉDIONI $(* * *)$, G. PORTAL $(* * *)$
}

(Manuscrit reçu le 4 mai 1976)

\begin{abstract}
RÉSUMÉ
On a calculé le kerma dans quelques matériaux radiothermoluminescents pour les neutrons d'énergie comprise entre 0,01 et $18 \mathrm{MeV}$. Les matériaux RTL étudiés sont ceux que l'on utilise couramment dans nos laboratoires : ${ }^{6} \mathrm{LiF}$, ${ }^{n a t} \mathrm{LiF},{ }^{7} \mathrm{LiF}, \mathrm{CaSO}_{4}, \mathrm{Al}_{2} \mathrm{O}_{3}$ et $\mathrm{BeO}$. $\mathrm{La}$ comparaison des résultats obtenus avec ceux publiés pour le tissu mou humain montre que l'énergie transmise aux matériaux thermoluminescents est généralement beaucoup plus faible que celle transmise au tissu. Il n'en est pas de même pour les photons. Ceci explique en partie pourquoi la réponse relative des matériaux RTL aux neutrons rapides est faible par rapport à celle obtenue avec des rayonnements électromagnétiques.
\end{abstract}

\begin{abstract}
Calculations of kerma for neutrons in the energy range $0.01-18 \mathrm{MeV}$ have been carried out for several commonly used thermoluminescent materials, i. e. ${ }^{6} \mathrm{LiF},{ }^{\text {nat }} \mathrm{LiF},{ }^{7} \mathrm{LiF}, \mathrm{BeO}, \mathrm{CaSO}_{4}, \mathrm{Al}_{2} \mathrm{O}_{3}$. The values obtained are compared with the kerma in tissue. Kerma in TLD materials is generally much smaller than in tissue; this is one of the reasons for the low relative response of the studied TLD materials to fast neutrons as compared to gamma rays.
\end{abstract}

(*) Cette étude a été effectuée dans le cadre de la collaboration entre le Laboratoire de Dosimétrie radiologique de Prague et la Section technique d'Instrumentation et de Dosimétrie du Service technique d'Études de Protection du Commissariat à l'Énergie atomique. Prague.

(**) Laboratoire de Dosimétrie radiologique. Académie des Sciences de Tchécoslovaquie,

(***) Département de Protection. Service technique d'Études de Protection. Section technique d'Instrumentation et de Dosimétrie. Commissariat à l'Énergie atomique. B.P. $n^{\circ} 6$, 92260 Fontenay-aux-Roses.

RADIOPROTECTION, VOL. $11-\mathrm{N}^{\circ} 3$ 


\section{INTRODUCTION}

Il est bien connu que la réponse aux neutrons rapides des matériaux radiothermoluminescents couramment utilisés est faible comparée à celle qu'ils présentent vis-à-vis du rayonnement électromagnétique [1, 2].

La réponse relative $\mathscr{R}$, normalisée à l'énergie absorbée dans le tissu mou humain, peut être définie par la relation suivante :

$$
\mathscr{R}=\frac{T_{n}}{T_{\gamma}} \frac{\left(E_{\mathrm{abs}}\right)_{n}^{\mathrm{RTL}} /\left(E_{\mathrm{abs}}\right)_{n}^{\text {tissu }}}{\left(E_{\mathrm{abs}}\right)_{\gamma}^{\mathrm{RTL}} /\left(E_{\mathrm{abs}}\right)_{\gamma}^{\text {tissu }}},
$$

avec $T_{n}$ et $T_{\gamma}$, rendements de radiothermoluminescence pour les neutrons et les $\gamma ;\left(E_{\mathrm{abs}}\right)_{n}^{\mathrm{RTL}}$ et $\left(E_{\mathrm{abs}}\right)_{n}^{\text {tissu, }}$, énergie absorbée due aux neutrons dans le matériau RTL et dans le tissu; $\left(E_{\mathrm{abs}}\right)_{\gamma}^{\mathrm{RTL}}$ et $\left(E_{\mathrm{abs}}\right)_{\gamma}^{\text {tissu }}$, énergie absorbée due aux $\gamma$ dans le matériau RTL et dans le tissu.

Pour les matériaux étudiés ici les valeurs de $\left(E_{\mathrm{abs}}\right)_{\mathrm{\gamma}}^{\mathrm{RTL}}$ et de $\left(E_{\mathrm{abs}}\right)_{\gamma}^{\text {tissu }}$ sont très proches l'une de l'autre [3]. La faible valeur de leur réponse aux neutrons rapides peut donc être la conséquence du fait que

$$
T_{n}<T_{\gamma} \quad \text { et } / \text { ou } \quad\left(E_{\text {abs }}\right)_{n}^{\mathrm{RTL}}<\left(E_{\mathrm{abs}}\right)_{n}^{\text {tissu }} .
$$

Dans ce rapport, nous analysons la contribution du second facteur. Nous calculons l'énergie absorbée dans les matériaux radiothermoluminescents couramment utilisés ( $\left.{ }^{6} \mathrm{LiF},{ }^{7} \mathrm{LiF},{ }^{\text {nat }} \mathrm{LiF}, \mathrm{CaSO}_{4}: \mathrm{Dy}, \mathrm{Al}_{2} \mathrm{O}_{3}, \mathrm{BeO}\right)$ pour les neutrons d'énergie comprise entre 0,01 et $18 \mathrm{MeV}$ et nous la comparons à l'énergie absorbée dans les tissus mous humains.

\section{CALCUL DE L'ÉNERGIE TRANSMISE DANS UN MILIEU DONNÉ. RAPPELS THÉORIQUES}

Le kerma, énergie des neutrons transmise à un milieu donné sous forme d'énergie cinétique des particules secondaires chargées, est donné par la relation suivante [4] valable pour une fluence unitaire

$$
K\left(E_{n}\right)=\sum_{l} N_{l} \sum_{j} \sum_{i} \overline{\varepsilon_{l j i}\left(E_{n}\right)} \sigma_{l j}\left(E_{n}\right),
$$

où $N_{l}$ est le nombre de noyaux du type $l$ dans $1 \mathrm{~g}$ de matière; $\overline{\varepsilon_{l j i}\left(E_{n}\right)}$ représente l'énergie cinétique moyenne de la particule chargée du type $i$ formée lors d'une réaction neutronique du type $j$ sur un noyau du type $l ; \sigma_{l j}\left(E_{n}\right)$ est la section efficace de cette réaction; $E_{n}$ est l'énergie des neutrons.

La liste des réactions que nous avons prises en considération est présentée dans le tableau I. Les données neutroniques utilisées pour le calcul du kerma sont extraites des références bibliographiques [5] et [6]. 
Pour chaque type de réaction nous avons obtenu les valeurs de $\overline{\varepsilon_{l j i}\left(E_{n}\right)}$ et de $\sigma_{l j}\left(E_{n}\right)$ de la façon suivante :

\section{Diffusion élastique}

Pour les réactions de diffusion élastique, les valeurs des $\sigma_{l j}$ sont extraites de la référence [5] sauf celles relatives au ${ }^{19} \mathrm{~F}$ qui ont été trouvées dans la référence [6]. Quant au ${ }^{32} \mathrm{~S}$, les valeurs des sections efficaces sont prises des travaux [7] et [8]; pour les gammes d'énergie où ces valeurs n'étaient pas précisées nous avons supposé que :

- pour $E_{n}<2,4 \mathrm{MeV} \quad \sigma_{\text {élastique }}=\sigma_{\text {totale }}$;

- pour $E_{n}>5 \mathrm{MeV} \quad \sigma_{\text {élastique }}=1 / 2 \sigma_{\text {totale }}$.

Pour ce type de diffusion, l'énergie cinétique moyenne d'un noyau de recul est égale [4] à

$$
\overline{\varepsilon_{l j i}\left(E_{n}\right)}=\frac{2 M_{n} M_{i}}{\left(M_{n}+M_{i}\right)^{2}} E_{n}\left(1-\overline{\cos \theta_{c m}}\right),
$$

où $M_{n}$ et $M_{i}$ sont respectivement les masses du neutron et du noyau de recul; $\theta_{c m}$ est l'angle de diffusion dans le système de centre de masse.

Les valeurs de $\overline{\cos \theta_{c m}}$ sont données par les références [5] et [6] sauf celles $\mathrm{du}{ }^{32} \mathrm{~S}$ qui sont interpolées à partir des données relatives aux ${ }^{19} \mathrm{~F},{ }^{27} \mathrm{Al}$ et ${ }^{40} \mathrm{Ca}$.

\section{Diffusion inélastique}

Les valeurs $\sigma_{l j}\left(E_{n}\right)$ des diffusions inélastiques sont extraites comme précédemment des références [5] et [6]. Toutefois, en ce qui concerne le ${ }^{32} \mathrm{~S}$ on a soustrait à la section efficace totale les sections efficaces des autres réactions prises en considération. Les différents paramètres sont tirés des références [7] et [8] sauf ceux correspondant à la réaction ${ }^{32} \mathrm{~S}(n, p){ }^{32} \mathrm{P}$ que l'on a trouvés dans la référence [9].

L'énergie moyenne du noyau de recul a été calculée à partir de la relation

$$
\overline{\varepsilon_{l j i}\left(E_{n}\right)}=\frac{2 M_{n} M_{i}}{\left(M_{n}+M_{i}\right)^{2}}\left(E_{n}+Q\right),
$$

en supposant la diffusion inélastique isotrope dans le système du centre de masse. $Q$ représente l'énergie d'excitation du noyau.

\section{Autres types de réactions}

Les valeurs de $\sigma_{l j}\left(E_{n}\right)$ des autres types de réactions ont été obtenues de la même façon que précédemment sauf celles relatives aux réactions ${ }^{19} \mathrm{~F}(n, p)$ et ${ }^{19} \mathrm{~F}(n, \alpha)$ pour lesquelles on a procédé à une interpolation linéaire des résultats expérimentaux des références [10] à [14]. 
TABLEAU I

Liste DES RÉACTIONS PRISES EN CONSIDÉRATION POUR LE CALCUL DES $K\left(E_{n}\right)$

\begin{tabular}{|c|c|c|c|c|c|}
\hline Noyau & Réaction & $\begin{array}{c}\mathrm{Q} \\
(\mathrm{MeV})\end{array}$ & Noyau & Réaction & $\begin{array}{c}\mathrm{Q} \\
(\mathrm{MeV})\end{array}$ \\
\hline${ }^{6} \mathrm{Li}$ & $\begin{array}{l}\text { diffusion } \\
\text { élastique } \\
\text { diffusion } \\
\text { inélastique } \\
(\mathrm{n} ; \mathrm{n}, \mathrm{d}) \\
(\mathrm{n} ; 2 \mathrm{n}, \mathrm{p}) \\
(\mathrm{n} ; \mathrm{p}) \\
(\mathrm{n} ; \alpha)\end{array}$ & $\begin{array}{l}-3,560 \\
-\quad 1,471 \\
-\quad 3,696 \\
-\quad 2,733 \\
+\quad 4,786\end{array}$ & ${ }^{7} \mathrm{Li}$ & $\begin{array}{l}\text { diffusion } \\
\text { élastique } \\
\text { diffusion } \\
\text { inélastique } \\
(n ; n ', t) \\
(n ; 2 n) \\
(n ; 2 n, d) \\
(n ; d)\end{array}$ & $\begin{array}{l}-\quad 0,478 \\
-\quad 2,466 \\
-\quad 7,251 \\
-8,723 \\
-\quad 7,760\end{array}$ \\
\hline${ }^{9} \mathrm{Be}$ & $\begin{array}{l}\text { diffusion } \\
\text { élastique } \\
(\mathrm{n} ; 2 \mathrm{n}) \\
(\mathrm{n} ; \mathrm{p}) \\
(\mathrm{n} ; \mathrm{d}) \\
(\mathrm{n} ; \mathrm{t}) \\
(\mathrm{n} ; \alpha)\end{array}$ & $\begin{array}{c}-1,66 ;-4,09 \\
-8,42 \\
-12,83 \\
-14,66 \\
-10,43 \\
-0,60\end{array}$ & ${ }^{16} \mathrm{O}$ & $\begin{array}{l}\begin{array}{l}\text { diffusion } \\
\text { élastique } \\
\text { diffusion } \\
\text { inélastique }\end{array} \\
(\mathrm{n} ; \mathrm{p}) \\
(\mathrm{n} ; \mathrm{d}) \\
(\mathrm{n} ; \alpha)\end{array}$ & $\begin{array}{c}\mathrm{de}-6,052 \mathrm{a} \\
-16,75 ; \\
33 \text { états excités } \\
-9,639 \\
-9,901 \\
-2,215 ;-5,899 \\
-6,068\end{array}$ \\
\hline${ }^{19} \mathrm{~F}$ & $\begin{array}{l}\text { diffusion } \\
\text { élastique } \\
\text { diffusion } \\
\text { inélastique } \\
(\mathrm{n} ; 2 \mathrm{n}) \\
(\mathrm{n} ; \mathrm{p}) \\
(\mathrm{n} ; \alpha)\end{array}$ & $\begin{array}{l}-\quad 0,109 \\
-10,43 \\
-\quad 4,00 \\
-\quad 1,50\end{array}$ & ${ }^{27} \mathrm{Al}$ & $\begin{array}{l}\begin{array}{l}\text { diffusion } \\
\text { élastique } \\
\text { diffusion } \\
\text { inélastique }\end{array} \\
(\mathrm{n} ; \mathrm{p}) \\
(\mathrm{n} ; \mathrm{d}) \\
(\mathrm{n} ; \mathrm{t}) \\
(\mathrm{n} ; \alpha)\end{array}$ & $\begin{array}{c}\text { de }-0,926 \text { à } \\
\text { - } 16,75 ; \\
37 \text { états excités } \\
-1,831 \\
-\quad 6,046 \\
-10,88 \\
-\quad 3,131\end{array}$ \\
\hline${ }^{32} \mathrm{~s}$ & $\begin{array}{l}\text { diffusion } \\
\text { élastique } \\
\text { diffusion } \\
\text { inélastique } \\
(\mathrm{n} ; \mathrm{p}) \\
(\mathrm{n} ; \alpha)\end{array}$ & $\begin{array}{l}-2,24 \\
-\quad 0,93 \\
+\quad 1,53\end{array}$ & ${ }^{40} \mathrm{Ca}$ & $\begin{array}{l}\begin{array}{l}\text { diffusion } \\
\text { élastique } \\
\text { diffusion } \\
\text { inélastique }\end{array} \\
\left(\mathrm{n} ; \mathrm{n}^{\prime}, \mathrm{p}\right) \\
(\mathrm{n} ; \mathrm{n}, \alpha) \\
(\mathrm{n} ; \mathrm{p}) \\
(\mathrm{n} ; \alpha)\end{array}$ & $\begin{array}{l}\text { de }-1,58 \text { à } \\
-8,775 ; \\
25 \text { états excités } \\
-8,34 \\
-\quad 7,04 \\
-0,532 \\
+1,653\end{array}$ \\
\hline
\end{tabular}

L'énergie cinétique moyenne d'une particule chargée issue de telles réactions a été calculée en supposant son émission isotrope dans le système du centre de masse. Par exemple, pour la réaction :

$$
n+l \rightarrow i+i^{\prime},
$$




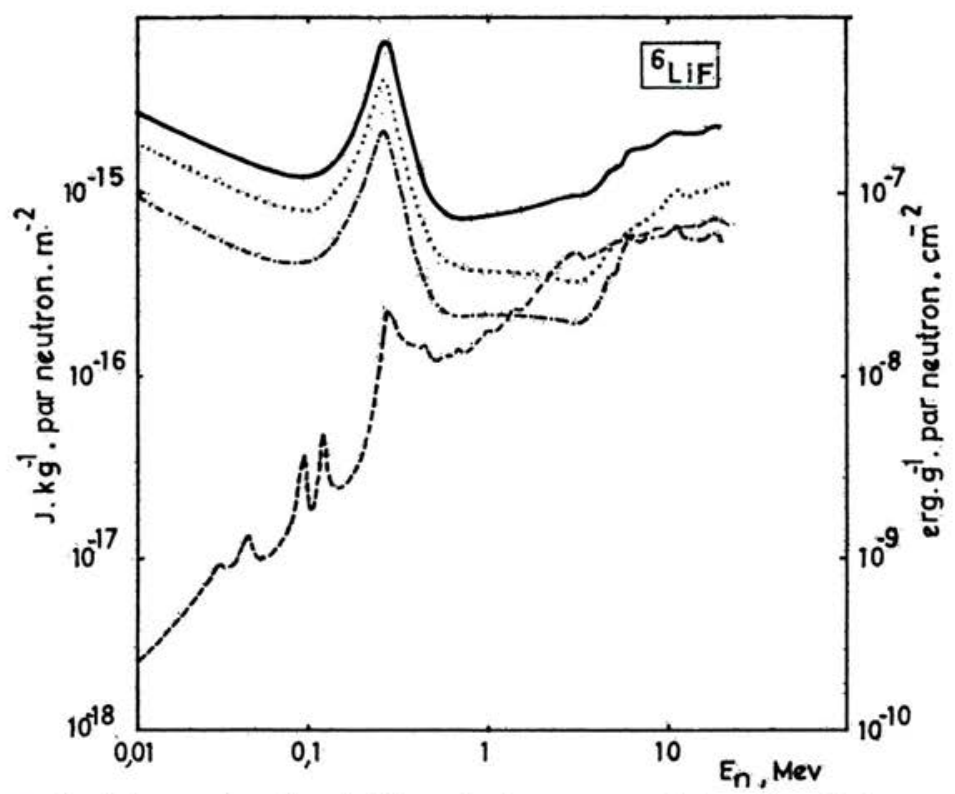

Fig. 1. - Variation en fonction de l'énergie des neutrons de la valeur du kerma pour une fluence unitaire pour le fluorure de lithium 6 :

$\longrightarrow$ total;

.... - dû aux noyaux de recul;

-...- dû aux noyaux d'hélium;

....... dû aux noyaux d'hydrogène.

on a la relation [4] :

$$
\overline{\varepsilon_{l j i}\left(E_{n}\right)}=\frac{M_{n} M_{i}+M_{l} M_{i^{\prime}}}{\left(M_{n}+M_{l}\right)\left(M_{i}+M_{i^{\prime}}\right)} E_{n}+\frac{M_{i^{\prime}}}{M_{n}+M_{l}} Q
$$

où $M_{l}$ et $M_{i^{\prime}}$ sont respectivement les masses du noyau cible et de la particule de type $i^{\prime}$ émise; $Q$ est, soit le bilan énergétique de la réaction, soit la somme de ce bilan et de l'énergie d'excitation du noyau formé.

Pour les réactions qui provoquent l'émission de plusieurs particules (voir le tableau I), on a trouvé la distribution énergétique des neutrons secondaires dans la référence [6]. Le calcul des énergies cinétiques moyennes des particules chargées lourdes a été effectué dans le système du centre de masse en supposant l'existence d'un noyau composé.

\section{RÉSULTATS ET DISCUSSIONS}

\section{III.1. Calcul du kerma partiel ou total}

Le calcul de $K\left(E_{n}\right)$ a été effectué pour les énergies $E_{n}$ de neutrons étudiés par BACH et CASWELL et mentionnées dans le rapport n ${ }^{\circ} 13$ de l'I.C.R.U. [15].

vol. $11-\mathrm{N}^{\circ} 3$ 
Dans les figures 1 à 6 on présente les résultats obtenus pour les noyaux d'hydrogène, les noyaux d'hélium, les autres noyaux de recul et pour l'ensemble de ces noyaux (kerma total). Les valeurs calculées pour le kerma total sont généralement en bon accord avec celles obtenues par d'autres auteurs pour les fluorures de lithium, le sulfate de calcium et l'oxyde de béryllium $[16,17,18]$. Remarquons que les travaux cités ne donnent pas les valeurs des kermas partiels.

Analysons les résultats pour chaque matériau étudié.

\section{Le fluorure de lithium 6 (fig. 1)}

Dans le cas du fluorure de lithium 6 les réactions sur le ${ }^{6} \mathrm{Li}$ prédominent largement.

Pour les neutrons d'énergie inférieure à $0,5 \mathrm{MeV}$ la réaction : ${ }^{6} \mathrm{Li}(n, \alpha)^{3} \mathrm{H}$ représente $90 \mathrm{p}$. cent du kerma total; pour les énergies inférieures à $3,8 \mathrm{MeV}$ elle y contribue pour plus de $50 \mathrm{p}$. cent; c'est elle qui est responsable du pic qui apparaît à $250 \mathrm{keV}$.

Pour les énergies supérieures à $5 \mathrm{MeV}$ l'importance des autres réactions croît et tout particulièrement celle de la réaction ${ }^{6} \mathrm{Li}\left(n ; n^{\prime}, d\right)^{4} \mathrm{He}$.

On constate qu'au-delà du domaine d'énergie où prédomine la réaction ${ }^{6} \mathrm{Li}(n, \alpha){ }^{3} \mathrm{H}$, les trois types de particules présentent des contributions comparables. Rappelons qu'en ce qui concerne les noyaux de recul ce sont les noyaux de ${ }^{6} \mathrm{Li}$ qui jouent un rôle majeur.

\section{Les fluorures de lithium 7 et de lithium naturel ( fig. 2 et 3)}

La réaction ${ }^{6} \mathrm{Li}(n, \alpha){ }^{3} \mathrm{H}$ représente plus de $50 \mathrm{p}$. cent du kerma total pour les énergies $E_{n}$ inférieures à $0,35 \mathrm{MeV}$. Pour les énergies supérieures à cette limite le fluorure de lithium 7 et le fluorure de lithium naturel présentent pratiquement les mêmes réactions.

Il s'agit essentiellement, pour $E_{n}<4 \mathrm{MeV}$, de la diffusion élastique sur le lithium 7 et, pour $E_{n} \geqq 7 \mathrm{MeV}$, de la réaction ${ }^{7} \mathrm{Li}\left(n ; n^{\prime}, t\right)^{4} \mathrm{He}$.

Contrairement à ce qui se passe pour le ${ }^{6} \mathrm{LiF}$, les réactions sur le ${ }^{19} \mathrm{~F}$ ne sont pas négligeables; la diffusion élastique est responsable des maximums qui apparaissent à $0,1 \mathrm{MeV}$ environ et la diffusion inélastique est importante aux énergies supérieures à $10 \mathrm{MeV}$.

Il apparaît clairement sur les figures 2 et 3 que les noyaux de recul jouent un rôle prépondérant pour les énergies de neutrons supérieures à $0,3 \mathrm{MeV}$ dans le cas du nat LiF et pour toute la gamme d'énergie étudiée dans celui du ${ }^{7} \mathrm{LiF}$.

\section{Le sulfate de calcium et l'oxyde d'aluminium (fig. 4 et 5)}

L'allure des 2 ensembles de courbes est comparable.

Pour les énergies inférieures à $2 \mathrm{MeV}$ sur la figure 4 et $4 \mathrm{MeV}$ sur la figure 5, seule la diffusion élastique est importante. L'oxygène 16 est responsable des nombreux pics. 


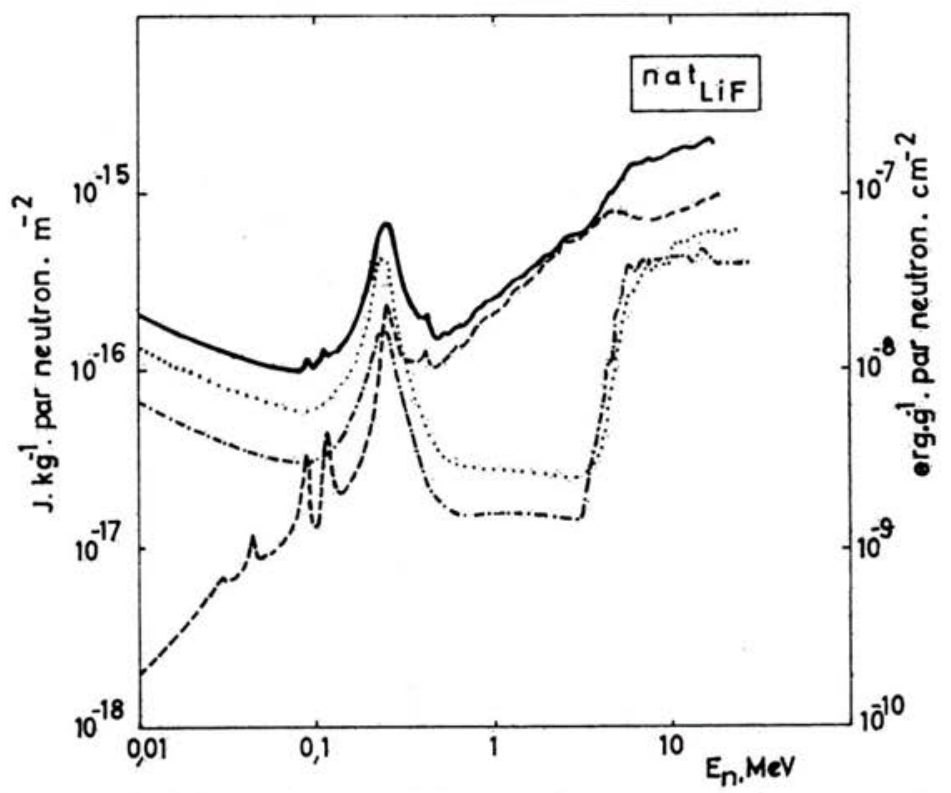

FIG. 2. - Variation en fonction de l'énergie des neutrons de la valeur du kerma pour le fluorure de lithium naturel.

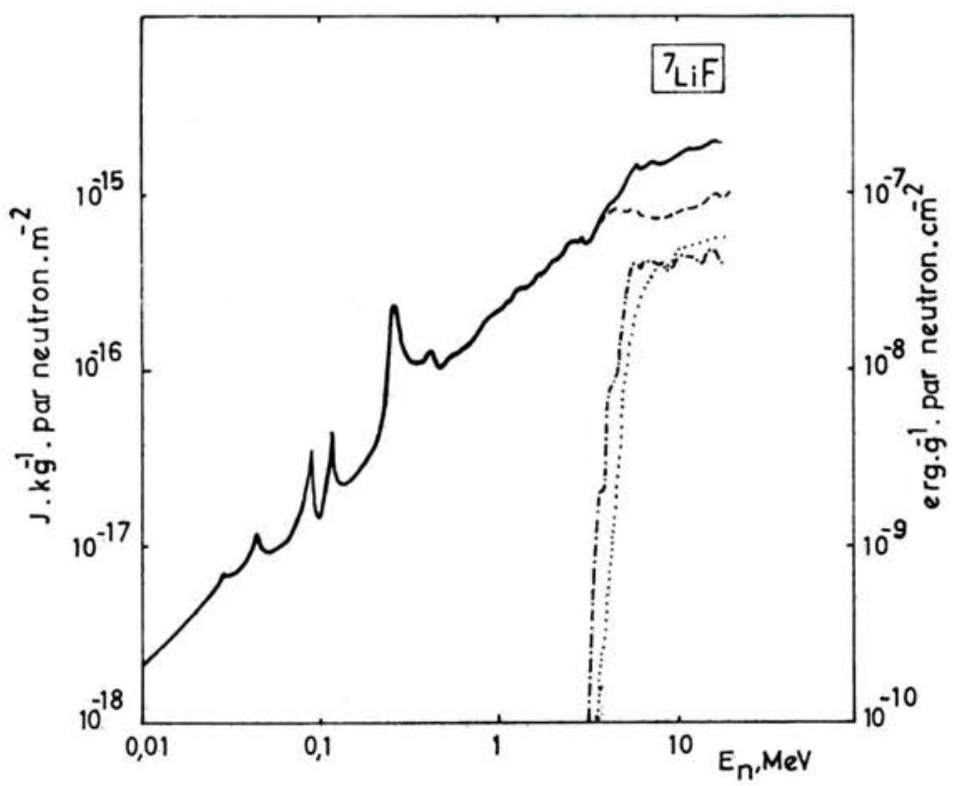

Fig. 3. - Variation en fonction de l'énergie des neutrons de la valeur du kerma pour le fluorure de lithium 7. 


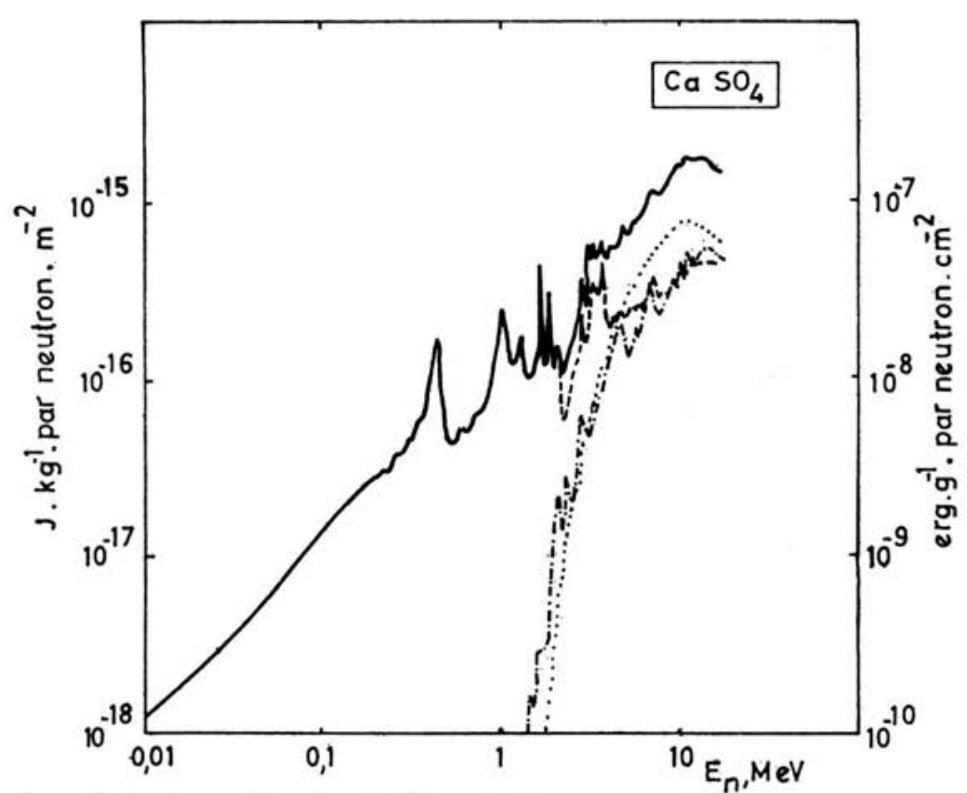

Fig. 4. - Variation en fonction de l'énergie des neutrons de la valeur du kerma pour le sulfate de calcium.

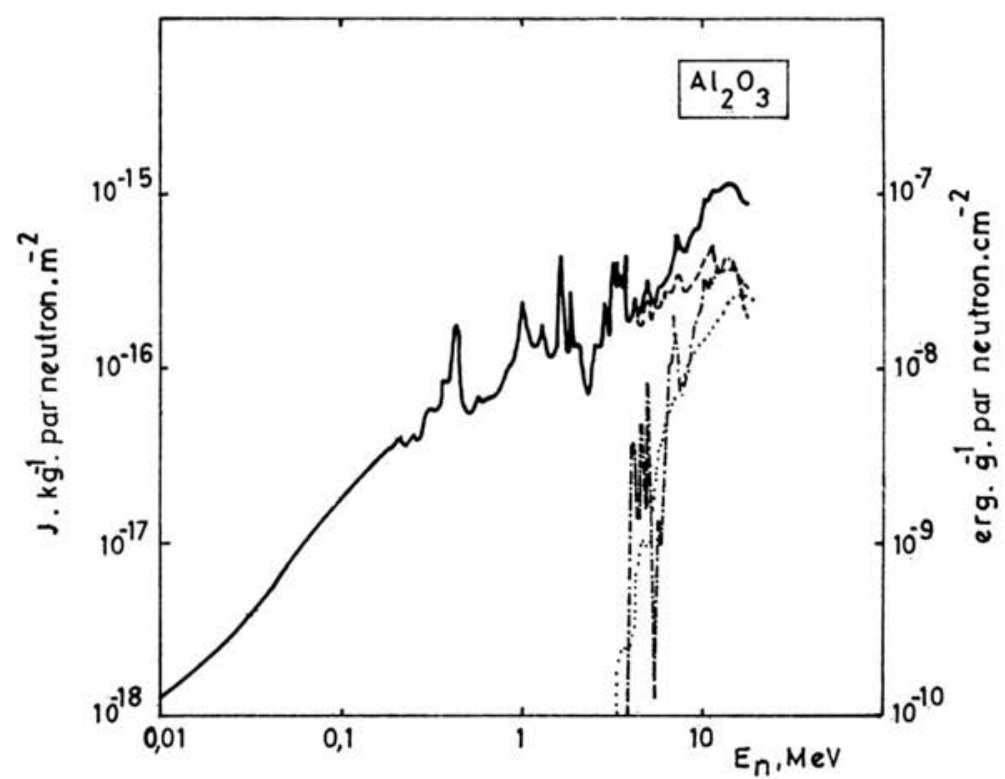

FIG. 5. - Variation en fonction de l'énergie des neutrons de la valeur du kerma pour l'alumine. 
Aux énergies supérieures à ces limites, d'autres réactions interviennent; ce sont essentiellement :

- pour le sulfate de calcium :

${ }^{16} \mathrm{O}(n, \alpha){ }^{13} \mathrm{C},{ }^{32} \mathrm{~S}(n, p){ }^{32} \mathrm{P},{ }^{32} \mathrm{~S}(n, \alpha){ }^{29} \mathrm{Si},{ }^{40} \mathrm{Ca}(n, p){ }^{40} \mathrm{~K},{ }^{40} \mathrm{Ca}(n, \alpha){ }^{37} \mathrm{Ar} ;$

- pour l'alumine :

$$
{ }^{16} \mathrm{O}(n, \alpha){ }^{13} \mathrm{C},{ }^{27} \mathrm{Al}(n, p){ }^{27} \mathrm{Mg},{ }^{27} \mathrm{Al}(n, \alpha){ }^{24} \mathrm{Na} .
$$

Il faut également remarquer que pour les neutrons d'énergie supérieure à $5 \mathrm{MeV}$ la contribution de la diffusion inélastique sur ${ }^{27} \mathrm{Al}$ est importante.

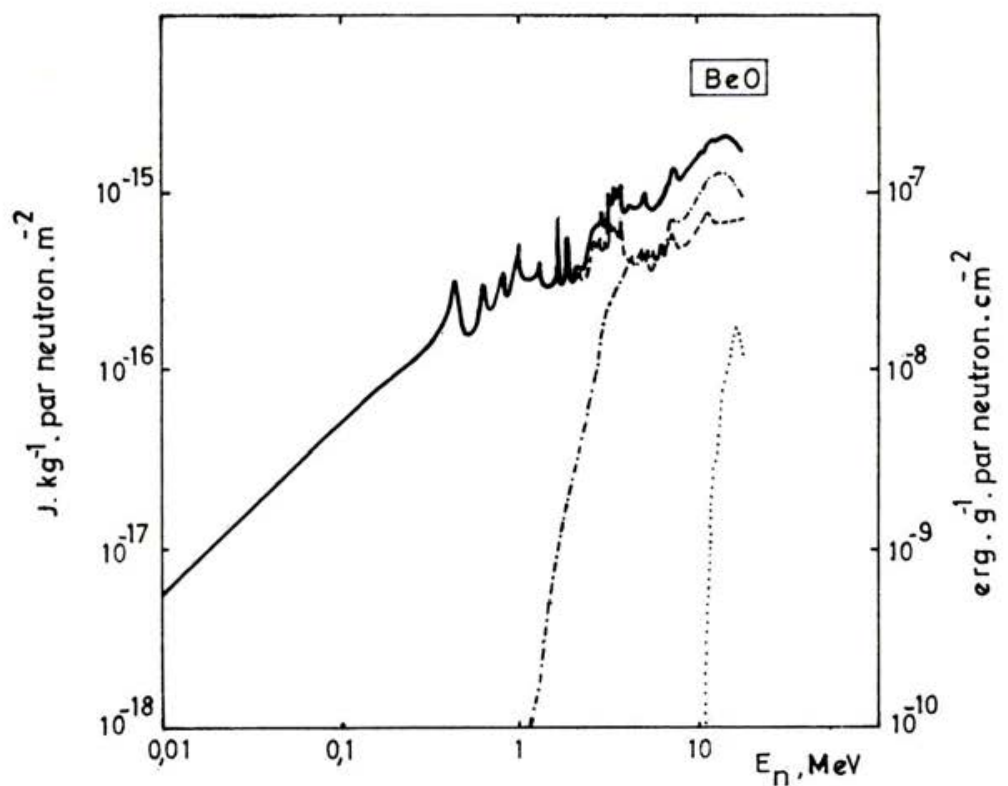

FIG. 6. - Variation en fonction de l'énergie des neutrons de la valeur du kerma pour l'oxyde de béryllium.

On constate sur les figures 4 et 5 la participation importante des noyaux de recul jusqu'à $4 \mathrm{MeV}$ pour le $\mathrm{SO}_{4} \mathrm{Ca}$ et jusqu'à $10 \mathrm{MeV}$ pour l'alumine. La limite est plus basse pour le sulfate de calcium que pour l'alumine car les réactions ${ }^{40} \mathrm{Ca}(n, p)$ et ${ }^{40} \mathrm{Ca}(n, \alpha)$ ont des sections efficaces importantes et présentent des valeurs de $Q$ assez faibles (voir tableau I).

L'oxyde de béryllium (fig. 6)

Jusqu'à $2 \mathrm{MeV}$ la forme des courbes de la figure 6 est comparable à celle des figures 4 et 5 . On constate néanmoins que les maximums corres- 
pondant aux résonances de la diffusion élastique sur ${ }^{16} \mathrm{O}$ sont moins accentués que pour l'alumine et le sulfate de calcium. Ceci provient du fait que la diffusion élastique sur le ${ }^{9} \mathrm{Be}$ est aussi importante que celle produite sur ${ }^{16} \mathrm{O}$.

On note également l'importance des noyaux d'hélium pour les énergies supérieures à quelques $\mathrm{MeV}$. Ceci provient du fait que les réactions sur le ${ }^{9} \mathrm{Be}$ sont susceptibles de produire deux particules $\alpha$; c'est le cas de la réaction ${ }^{9} \mathrm{Be}(n, 2 n) 2 \alpha$ dont la contribution représente plus de $30 \mathrm{p}$. cent du kerma total pour les énergies supérieures à $4 \mathrm{MeV}$.

Les noyaux d'hydrogène ne jouent qu'un rôle très faible.

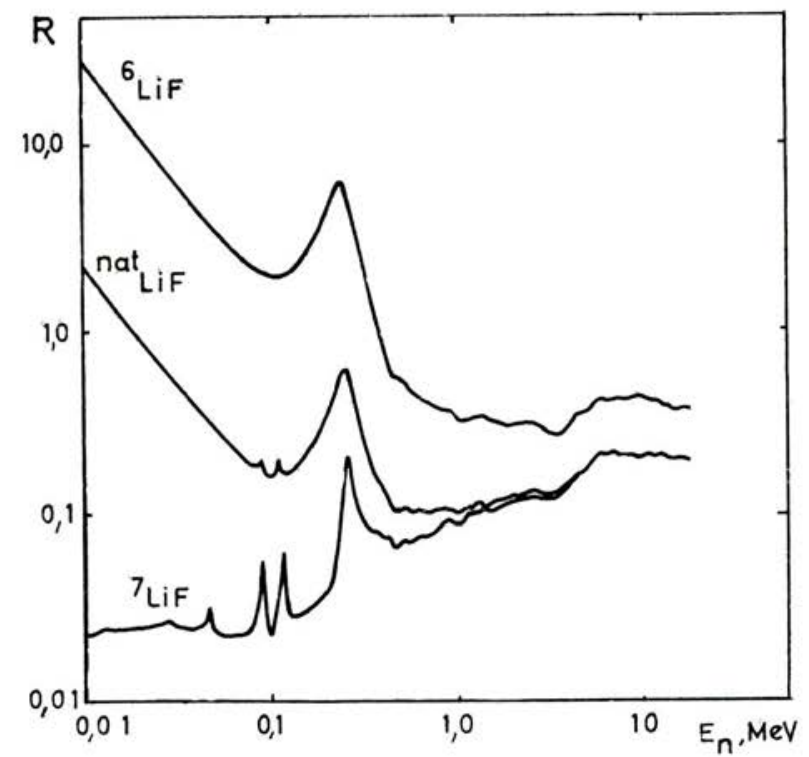

FIG. 7. - Variation en fonction de l'énergie des neutrons du rapport $\mathrm{R}$ du kerma total dans les fluorures de lithium (6,7 et naturel) et dans le tissu.

\section{Synthèse des résultats}

$\mathrm{Si}$ on compare les valeurs des facteurs de conversion «fluence-kerma total » des figures 1 à 6 on constate que :

- pour les matériaux ne contenant pas de ${ }^{6} \mathrm{Li}$ les facteurs de conversion croissent avec l'énergie jusqu'à un seuil de saturation qui est voisin de $10 \mathrm{MeV}$.

L'oxyde de béryllium présente les plus fortes valeurs, l'alumine présente les plus faibles. Celles du fluorure de lithium 7 et du sulfate de calcium sont voisines de celles de l'alumine aux faibles énergies et de celles de l'oxyde de béryllium aux fortes énergies :

- pour les matériaux contenant $\mathrm{du}{ }^{6} \mathrm{Li}$ on remarque aux énergies inférieures à $1 \mathrm{MeV}$ l'influence de la réaction ${ }^{6} \mathrm{Li}(n, \alpha)^{3} \mathrm{H}$; si l'on exclut le pic 
situé à $250 \mathrm{keV}$, le facteur de conversion varie peu avec l'énergie des neutrons entre $10 \mathrm{keV}$ et $1 \mathrm{MeV}$.

\section{2. COMPaRAison AVEC LE TISSU MOU hUmain}

Dans les figures 7 et 8 nous comparons ces facteurs de conversion "fluence-kerma total» avec ceux obtenus pour le tissu mou par BACH et CASWEll et publiés dans le rapport $\mathrm{n}^{\circ} 13$ de l'I.C.R.U. [15].

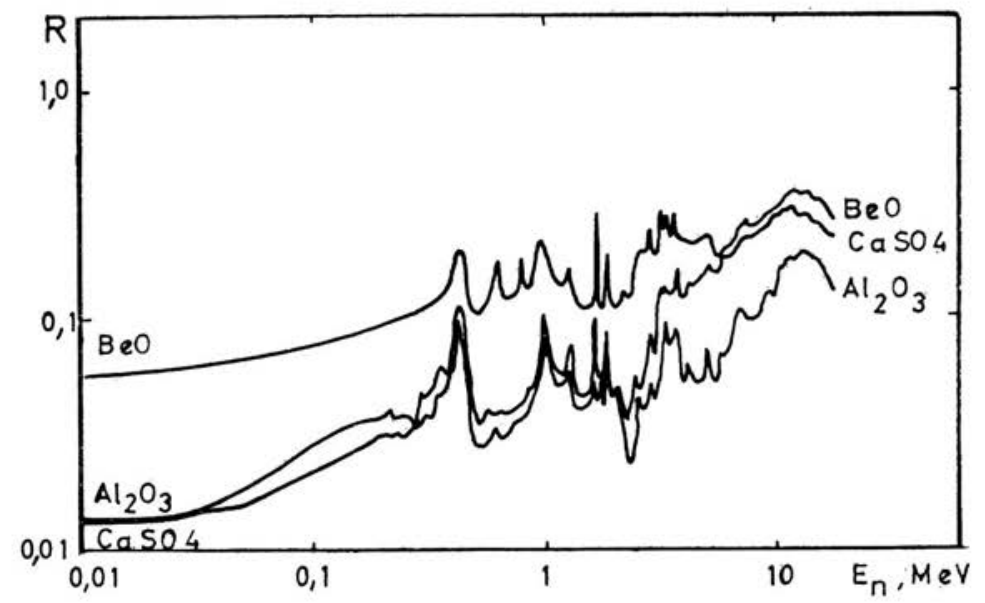

FIG. 8. - Variation en fonction de l'énergie des neutrons du rapport $\mathrm{R}$ du kerma total dans le sulfate de calcium (alumine ou oxyde de béryllium) et dans le tissu.

La figure 7 donne les résultats pour les trois compositions isotopiques de fluorure de lithium. On remarque que l'énergie transmise à ces matériaux radiothermoluminescents est généralement plus faible que celle transmise au tissu (hormis le cas du ${ }^{6} \mathrm{LiF}$ pour $E_{n}<0,4 \mathrm{MeV}$ ).

Ce fait est particulièrement accusé pour le ${ }^{7} \mathrm{LiF}$; la différence avec le ${ }^{\text {nat }} \mathrm{LiF}$ est très importante dans le domaine des faibles énergies; elle est réduite à $20 \mathrm{p}$. cent environ à $1 \mathrm{MeV}$ et devient très faible (moins de $5 \mathrm{p}$. cent) au-delà de $3,8 \mathrm{MeV}$.

Les réponses du sulfate de calcium, de l'alumine et de l'oxyde de béryllium évoluent en fonction de l'énergie des neutrons d'une façon similaire à celle du fluorure de lithium 7 . Les valeurs relatives maximales ne dépassent pas 0,3 pour le sulfate de calcium et l'oxyde de béryllium et 0,2 pour l'alumine.

\section{III.3. CONCLUSION}

Ces résultats montrent que si, comme nous l'avons fait remarquer dans l'introduction, la réponse relative des matériaux radiothermoluminescents est

vOL. $11-\mathrm{N}^{\circ} 3$ 
faible, cela provient avant tout du fait que l'énergie absorbée dans ces matériaux est très inférieure à celle absorbée dans les tissus mous :

$$
\left(E_{\mathrm{abs}}\right)_{n}^{\mathrm{RTL}}<\left(E_{\mathrm{abs}}\right)_{n}^{\mathrm{tissu}} \quad(\text { équation } 2) .
$$

Cependant, si on compare les résultats de ces calculs à ceux de quelques expériences réalisées récemment [2] pour des neutrons de 14,7 MeV (tableau II),

TABLEAU II

COMPARAISON DES RAPPORTS DES KERMAS TOTAUX DANS LES MATÉRIAUX THERMOLUMINESCENTS et DANS LE TISSU MOU humain POUR DES NEUtRONS DE $14,7 \mathrm{MeV}$

\begin{tabular}{|c|c|c|}
\hline Matériau thermoluminescent & Valeurs expérimentales [2] & Valeurs calculées \\
\hline${ }^{n a t}{ }_{\mathrm{LiF}}$ & 0,12 & 0,29 \\
\hline $7_{\mathrm{LiF}}$ & 0,088 & 0,28 \\
\hline $\mathrm{CaSO}_{4}: \mathrm{Dy}$ & 0,087 & 0,24 \\
\hline $\mathrm{Al}_{2} \mathrm{O}_{3}$ & 0,15 & 0,17 \\
\hline
\end{tabular}

on constate que la réponse expérimentale est en général inférieure à celle prévue par le calcul (kerma total relatif). Tenant compte de la précision des valeurs expérimentales $( \pm 10 \mathrm{p}$. cent) et des valeurs théoriques $(<10 \mathrm{p}$. cent), ceci nous amène à conclure que le rendement de thermoluminescence après l'absorption de l'énergie libérée par les neutrons est, tout au moins dans le cas des $\mathrm{LiF}$ et $\mathrm{CaSO}_{4}$ : Dy, inférieur à celui correspondant à l'absorption de l'énergie libérée par les photons

$$
T_{n}<T_{\gamma} .
$$

On remarque également que l'importance de cette différence est variable avec le matériau considéré. Ce sont autant de phénomènes qu'il est nécessaire d'étudier si l'on désire être en mesure de calculer la réponse d'un matériau thermoluminescent quelconque irradié dans un champ de neutrons déterminés.

\section{BIBLIOGRAPHIE}

[1] Atrix F. H. Luminescence and exoelectron dosimetry in personnel monitoring. In Advances in physical and biological radiation detectors, Vienna, 23-27 November 1970. Vienna, IAEA, 1971, 3-15.

[2] Spurny F., Médioni R., Portal G. Les sensibilités de divers détecteurs thermoluminescents, photoluminescents et photographiques aux neutrons. In Proc. 2nd Symposium 
on neutron dosimetry in biology and medicine, Neuherberg 30 September-4 October 1974 (Burger G., éd.) Luxembourg, Communautés Européennes, EUR-5273 d-e-f, 1975, 1, 495-504.

[3] Portal G. Contribution à l'étude et au développement de la dosimétrie par thermoluminescence. Rapport CEA-R-4697, 1975.

[4] Bach R. L., Caswell R. S. Energy transfer to matter by neutrons. Radiat. Res., 1968, $35,1$.

[5] NNCSC. Evaluated Nuclear Data File ENDF/B. Version III. Prepared by the National Neutron Cross Section Center, Brookhaven National Laboratory, Upton, New York, 1970.

[6] United Kingdom Nuclear Data Library, AEE Winfrith.

[7] Stehn J. R., Goldberg M. D., Magurno B. A., Wiener-Chasman R. Neutron cross sections, BNL-325, 2nd edition, Suppl. $\mathrm{n}^{\circ}$ 2, Brookhaven, 1964.

[8] Hughes D. J., Schwartz R. B. Neutron cross section, BNL-325, 2nd edition, Brookhaven, 1958.

[9] Schett A., OKamoto K., Lesca L., Fröhner F. H., Liskien H., Paulsen A. Compilation of threshold reaction neutron cross sections for neutron dosimetry and other applications, CCDN, EANDC 95 " U », Saclay, 1974.

[10] Bass R., Haug P., Krüger K., Staginnus B. Fast neutron excitation functions by activation techniques. In Progress report on nuclear data research in the Euratom community. EANDC (E) 66 " U », NFA, Paris, 1966, 64.

[11] Bormann M., Fretwurst E., Schehka P., Wrege G., Büttner H., Lindner A., MELDNER $\mathrm{H}$. Some excitation functions of neutron induced reactions in the energy range 12.6-19.6 MeV. Nucl. Phys., 1965, 63, 438.

[12] Bostrom N. A., Hudspeth E. L., Morgan I. L. Excitation curve for $\mathrm{F}^{19}(n, \alpha) \mathrm{N}^{16}$. Phys. Rev., 1955, 99, 643.

[13] Sмith D. M., Bostrom N. A., Hudspeth E. L. Excitation study of $\mathrm{F}^{19}(n, \alpha) \mathrm{N}^{16}$ with a $\mathrm{BaF}_{2}$ crystal. Phys. Rev., 1960, 117, 514.

[14] Picard J., Williamson G. F. The $(n, p),(n, \alpha)$ and $(n, 2 n)$ cross sections for $\mathrm{F}^{19}$ and $\mathrm{Na}^{23}$ between 13 and $21 \mathrm{MeV}$. Nucl. Phys., 1965, 63, 673.

[15] I.C.R.U. Neutron fluence, neutron spectra and kerma. ICRU Report 13, Washington, 1969.

[16] Davy D. R., O'Brien B. G. An adapted model for the LET dependence of LiF thermoluminescence. Health Physics, 1969, 17, 471.

[17] Furuta Y., TANAKA S. Response of ${ }^{6} \mathrm{LiF}$ and ${ }^{7} \mathrm{LiF}$ thermoluminescence dosimeters to fast neutrons. Nucl. Instr. Meth., 1972, 104, 365.

[18] TANAKA S., Furuta Y. Neutron responses of thermoluminescence dosimeters, $\mathrm{BeO}(\mathrm{Na})$, $\mathrm{CaSO}_{4}(\mathrm{Tm})$, and its mixture with ${ }^{6} \mathrm{LiF}$ or ${ }^{7} \mathrm{LiF}$. Proc. 4th Intern. Conference on luminescence dosimetry, 27-31 Aug., 1974 (T. Niewiadomski ed.) Krakow, Institute of nuclear physics, 1975, 3, 1213.

[19] SPURnÝ F., Portal G. Sensitivity of various RTL and RPL materials to thermal neutrons Proc. 4th Intern. Conference on luminescence dosimetry (T. Niewiadomski ed.) Krakow, Institute of nuclear physics, 1975, 3, 1105. 